\title{
Supplement of bamboo extract lowers serum monocyte chemoattractant protein-1 concentration in mice fed a diet containing a high level of saturated fat
}

\author{
Jason K. Higa, Wanyu Liu, Marla J. Berry and Jun Panee* \\ Department of Cell and Molecular Biology, John A. Burns School of Medicine, University of Hawaii at Manoa, 651 Ilalo Street \\ BSB 222, Honolulu, HI 968 13, USA \\ (Received 24 May 2010 - Revised 10 March 2011 - Accepted 22 March 2011 - First published online 7 June 2011)
}

\section{Abstract}

Monocyte chemoattractant protein-1 (MCP-1) is an inflammatory chemokine up-regulated in obese subjects, contributing to the development of type 2 diabetes. The present study investigated the inhibitory effect of an ethanol-water extract from bamboo (BEX, Phyllostachys edulis) on the blood concentration of MCP-1. C57BL/6J mice were fed a standard diet or a high-fat diet with or without the BEX supplement ( $11 \mathrm{~g}$ dry mass $/ 17000 \mathrm{~kJ})$ for 6 months. A total of ten mice were used in each group. Body weight and food consumption were measured weekly. After euthanisation, the weight of visceral fat and circulating MCP-1 concentration were measured. In comparison with the standard control group, the high-fat control group had increased body weight, abdominal fat storage and serum MCP-1 concentration by $60 \%(P<0.001), 266 \%(P<0.001)$ and $180 \%(P<0 \cdot 01)$, respectively. In comparison with the high-fat control group, the high-fat BEX group showed a $3 \%$ decrease in body weight $(P<0 \cdot 01), 24 \%$ decrease in mesenteric fat depot $(P<0 \cdot 01)$ and $49 \%$ decrease in serum MCP-1 concentration $(P<0 \cdot 05)$. The present study suggests that the BEX supplement in the high-fat diet ameliorates elevated MCP-1 concentrations in the blood, and whether this is related to modulated endocrine properties of the visceral fat is to be studied.

Key words: Monocyte chemoattractant protein-1: High-fat diet: Bamboo extract: Mesenteric fat

Monocyte chemoattractant protein-1 (MCP-1/chemokine (C-C motif) ligand 2) is a member of the $\mathrm{C}-\mathrm{C}$ chemotactic cytokine (chemokine) family, produced by multiple cell types constitutively or after induction ${ }^{(1)}$. The circulating level of MCP-1 was found to be approximately $50 \%$ higher in obese mice ${ }^{(2)}$ and in human subjects with type 2 diabetes ${ }^{(3)}$ in comparison with controls. MCP-1 recruits monocytes into adipose tissues and enhances obesity-associated chronic inflammation ${ }^{(4)}$ and insulin resistance ${ }^{(5)}$. It also facilitates the expansion and remodelling of the adipose tissue during the development of obesity through an angiogenic effect on the endothelial cells $^{(6)}$; furthermore, this chemokine can decrease the liposynthesis ability of adipocytes ${ }^{(2)}$ and subsequently elevate the NEFA level in the circulation ${ }^{(4)}$, exerting lipotoxicity in the periphery $^{(7)}$. Therefore, inhibiting MCP-1 overproduction has become a preventive strategy for obesity-induced type 2 diabetes.

Our previous study has shown that an ethanol-water extract from bamboo (BEX, Phyllostachys edulis) efficiently protected murine muscle $\mathrm{C} 2 \mathrm{C} 12$ cells from lipotoxicity ${ }^{(8)}$, an obesity-related condition leading to inflammation and insulin resistance ${ }^{(9,10)}$. In the present study, it is further revealed that the BEX as a dietary supplement significantly decreased the circulating level of MCP-1 in mice treated with a diet containing a high level of saturated fat, with concurrence of decreased weight of the mesenteric fat depot.

\section{Experimental methods}

\section{Bamboo extract}

The BEX used in the present study was provided by Golden Basin LLC (Kailua, HI, USA). It is prepared from fresh leaves and small branches of bamboo ( $P$. edulis) in Hunan Province, China, through a patented ethanol-water extraction procedure (Chinese invention patent, CN 1287848A).

\section{Animals}

Male C57BL/6J mice at 4 weeks of age were purchased from Jackson Laboratories (Bar Harbor, MN, USA). The animals were housed three to four per cage and had ad libitum access to water and food. The room temperature was controlled at $20^{\circ} \mathrm{C}$, and lighting was turned on and off with $12 \mathrm{~h}$

Abbreviations: BEX, bamboo extract; MCP-1, monocyte chemoattractant protein-1. 
intervals. Institutional and national guidelines for the care and use of animals were followed, and all experimental procedures involving animals were approved by the Institutional Animal Care and Use Committee at the University of Hawaii (Honolulu, HI, USA).

\section{Dietary treatment}

After 1 week of acclimatisation with regular rodent chow, mice were fed a standard diet $(10 \%$ energy from fat) or a high-fat diet ( $60 \%$ energy from fat) with or without the BEX supplement ( $11 \mathrm{~g}$ dry mass per $17000 \mathrm{~kJ}$ ) for 6 months. A total of ten mice were used in each dietary group. Body weight and food consumption were measured weekly. All diets were purchased from Research Diets (New Brunswick, NJ, USA). The dietary composition is listed in Table 1.

\section{Measurement of the abdominal fat pads}

After euthanisation, epididymal fat, perirenal fat and mesenteric fat were collected and weighed.

\section{Serum monocyte chemoattractant protein-1 quantification}

Blood was collected through cardiac puncture. MCP-1 concentrations in the sera were measured using a Cytometric Bead Array - Mouse MCP-1 Flex Set (BD Biosciences, Bedford, MA, USA).

\section{Statistical analysis}

Statistical analysis was performed by two-way ANOVA with Bonferroni's post hoc test using the software Prism 4.0a (GraphPad Software, Inc., La Jolla, CA, USA) and Stata 11.0
(StataCorp LP, College Station, TX, USA) (Table 2). The weekly record of body weight was analysed using linear regression with Huber correction and random-effects regression to account for multiple measurements per mouse. $P \leq 0.05$ was considered statistically significant.

\section{Results}

Table 2 summarises the major findings of the present study. During the 6 months of treatment, the high dietary fat content increased the daily energy intake by approximately $30 \%$ $(P<0 \cdot 0001)$. The BEX supplement did not affect the energy intake. The body weight of mice at both start and end points is shown. High-fat diets resulted in an average of $60 \%$ increase in body weight $(P<0.0001)$ at the end point. When the weekly record of the body weight (data not shown) was analysed, the BEX supplement in the high-fat diet was found to slightly decrease $(-3 \%, P<0.01)$ the weight gain of mice.

The high dietary fat content also increased the total weight of the abdominal fat by approximately threefold. When the weight of the individual fat depot was analysed, the BEX was found to increase the epididymal fat by $20 \%(0.37 \mathrm{~g}$, $P<0.05)$ and decrease the mesenteric fat by $24 \%(0.52 \mathrm{~g}$, $P<0 \cdot 01$ ), but did not affect the total weight of the visceral fat. An interaction between the fat content and the BEX was found to play an important role in regulating the weight of the mesenteric fat depot $(P<0 \cdot 01)$.

Most interestingly, the BEX supplement dramatically decreased high-fat, diet-induced elevated MCP-1 concentration in the serum $(-49 \%, P<0.05)$, and whether this is related to modulated endocrine properties of the visceral fat, especially the mesenteric fat, is to be studied.

Table 1. Composition of the diets used in the present study

\begin{tabular}{|c|c|c|c|c|c|c|c|c|}
\hline \multirow[t]{2}{*}{ Diets... } & \multicolumn{2}{|c|}{ Standard control } & \multicolumn{2}{|c|}{ Standard BEX } & \multicolumn{2}{|c|}{ High-fat control } & \multicolumn{2}{|c|}{ High-fat BEX } \\
\hline & g & $\mathrm{kJ}$ & g & $\mathrm{kJ}$ & g & $\mathrm{kJ}$ & g & $\mathrm{kJ}$ \\
\hline \multicolumn{9}{|l|}{ Ingredients } \\
\hline Casein, 80 mesh & 200 & 3349 & 200 & 3349 & 200 & 3349 & 200 & 3349 \\
\hline L-Cystine & 3 & 50 & 3 & 50 & 3 & 50 & 3 & 50 \\
\hline Maize starch & 315 & 5275 & 315 & 5275 & 0 & 0 & 0 & 0 \\
\hline Maltodextrin 10 & 35 & 586 & 35 & 586 & 125 & 2093 & 125 & 2093 \\
\hline Sucrose & 350 & 5862 & 350 & 5862 & $68 \cdot 8$ & 1151 & $68 \cdot 8$ & 1151 \\
\hline Cellulose, BW200 & 50 & 0 & 50 & 0 & 50 & 0 & 50 & 0 \\
\hline Soyabean oil & 25 & 942 & 25 & 942 & 25 & 942 & 25 & 942 \\
\hline Lard & 20 & 754 & 20 & 754 & 245 & 9232 & 245 & 9232 \\
\hline Mineral mix S10026 & 10 & 0 & 10 & 0 & 10 & 0 & 10 & 0 \\
\hline Dicalcium phosphate & 13 & 0 & 13 & 0 & 13 & 0 & 13 & 0 \\
\hline Calcium carbonate & 5.5 & 38 & 5.5 & 38 & 5.5 & 38 & $5 \cdot 5$ & 38 \\
\hline Potassium citrate, $1 \mathrm{H}_{2} \mathrm{O}$ & $16 \cdot 5$ & 0 & $16 \cdot 5$ & 0 & $16 \cdot 5$ & 0 & $16 \cdot 5$ & 0 \\
\hline Vitamin mix V10001 & 10 & 167 & 10 & 167 & 10 & 167 & 10 & 167 \\
\hline Choline bitartrate & 2 & 0 & 2 & 0 & 2 & 0 & 2 & 0 \\
\hline Bamboo extract (dry mass) & 0 & 0 & 11 & 0 & 0 & 0 & 11 & 0 \\
\hline Water from the bamboo extract & 0 & 0 & 11 & 0 & 0 & 0 & 11 & 0 \\
\hline FD\&C Yellow Dye no. 5 & 0.05 & 0 & 0.025 & 0 & 0 & 0 & 0.025 & 0 \\
\hline FD\&C Red Dye no. 40 & 0 & 0 & 0 & 0 & 0 & 0 & 0.025 & 0 \\
\hline FD\&C Blue Dye no. 1 & 0 & 0 & 0.025 & 0 & 0.05 & 0 & 0 & 0 \\
\hline Total & $1055 \cdot 1$ & 16986 & $1077 \cdot 1$ & 16986 & 773.9 & 16986 & 795.9 & 16986 \\
\hline
\end{tabular}

BEX, bamboo extract. 
Table 2. Energy intake, body weight, abdominal fat and serum monocyte chemoattractant protein-1 (MCP-1) concentration in mice

(Mean values and standard deviations)

\begin{tabular}{|c|c|c|c|c|c|c|c|c|}
\hline \multirow[t]{2}{*}{ Group... } & \multicolumn{2}{|c|}{ Standard control } & \multicolumn{2}{|c|}{ Standard BEX } & \multicolumn{2}{|c|}{ High-fat control } & \multicolumn{2}{|c|}{ High-fat BEX } \\
\hline & Mean & SD & Mean & SD & Mean & SD & Mean & SD \\
\hline Energy intake $(\mathrm{kJ} / \mathrm{d})^{\star}$ & $39 \cdot 4^{\mathrm{a}}$ & $4 \cdot 0$ & $37 \cdot 9^{a}$ & 4.5 & $50 \cdot 3^{\mathrm{b}}$ & $5 \cdot 3$ & $50 \cdot 9^{b}$ & $3 \cdot 6$ \\
\hline Body weight (g) (start) & $17 \cdot 8^{\mathrm{a}}$ & $2 \cdot 1$ & $18 \cdot 2^{\mathrm{a}}$ & 1.8 & $18 \cdot 0^{\mathrm{a}}$ & 0.9 & $18 \cdot 4^{\mathrm{a}}$ & $1 \cdot 1$ \\
\hline Body weight (g) (end) & $28 \cdot 7^{\mathrm{a}}$ & $3 \cdot 1$ & $28 \cdot 3^{a}$ & 2.5 & $47 \cdot 5^{\mathrm{b}}$ & $2 \cdot 2$ & $44 \cdot 6^{\mathrm{b}}$ & $2 \cdot 6$ \\
\hline Epididymal fat (g) & $0.65^{\mathrm{a}}$ & 0.26 & $0.78^{\mathrm{a}}$ & 0.38 & $1.84^{\mathrm{b}}$ & 0.24 & $2 \cdot 21^{\mathrm{c}}$ & 0.37 \\
\hline Perirenal fat $(\mathrm{g})$ & $0 \cdot 17^{\mathrm{a}}$ & 0.07 & $0.24^{\mathrm{a}}$ & 0.16 & $0.85^{\mathrm{b}}$ & 0.31 & $0.74^{\mathrm{b}}$ & 0.19 \\
\hline Mesenteric and omental fat ( $\mathrm{g}$ ) & $0.22^{\mathrm{a}}$ & 0.08 & $0.30^{\mathrm{a}}$ & 0.14 & $2 \cdot 17^{b}$ & 0.23 & $1.65^{\mathrm{c}}$ & 0.47 \\
\hline Total abdominal fat $(\mathrm{g})$ & $1.04^{a}$ & 0.38 & $1 \cdot 32^{\mathrm{a}}$ & 0.66 & $4.86^{b}$ & 0.58 & $4.69^{b}$ & 0.39 \\
\hline Serum MCP-1 (pg/ml) & $16 \cdot 26^{\mathrm{a}}$ & 6.58 & $19 \cdot 34^{\mathrm{a}}$ & 3.23 & $45 \cdot 78^{\mathrm{b}}$ & 9.56 & $23 \cdot 37^{a}$ & 1.75 \\
\hline
\end{tabular}

BEX, bamboo extract.

$a, b, c$ Mean values within a row with unlike superscript letters were significantly different $(P<0.05)$

* Energy intake is the average of the weekly measurements.

\section{Discussion}

Using the same diet containing a high level of saturated fat, Yu et al. ${ }^{(11)}$ treated $\mathrm{C} 57 \mathrm{BL} / 6$ mice for 3 months and compared the MCP-1 expression and secretion in four types of adipose tissues: mesenteric, epididymal, perirenal and subcutaneous. While the amounts of MCP-1 released by the epididymal, perirenal and subcutaneous fat depots were approximately the same, this level quadruplicated in the mesenteric fat. Mesenteric adipose tissue-conditioned medium also induced the highest degree of macrophage migration and stimulated pro-inflammatory cytokine production in macrophages. These findings indicate that in comparison with the other fat depots, the mesenteric fat tissue has a more pronounced role in obesity-associated inflammation. The present study showed that the BEX supplement in a high-fat diet decreased the weight of the mesenteric fat by $0.52 \mathrm{~g}$, and therefore it may attenuate MCP-1 secretion from this tissue and subsequently contribute to the decrease in MCP-1 in the circulation. Although the BEX increased the weight of the epididymal fat by $0.37 \mathrm{~g}$, this may not compensate the change caused by the mesenteric fat due to the dramatic difference between the MCP-1 secretion abilities of these two fat depots. This suggests that the BEX may alter the distribution of fat storage in the visceral adipose tissues and lower MCP-1 secretion as a final result. Although white adipose tissue is a major source of MCP-1 ${ }^{(2)}$, this chemokine is also produced in other tissues $^{(12-14)}$, and therefore a systematic study is needed to evaluate the tissue-specific effect of BEX.

Other natural products that inhibit the overproduction of MCP-1 in obese/diabetic rodents and in cell culture models include traditional Asian medicine ${ }^{(15-18)}$, extracts from herbs $^{(19)}$, spices ${ }^{(20,21)}$, fruits and vegetables ${ }^{(22-25)}$. Due to the use of different models and experimental procedures, and variable purity of the extracts, it is difficult to perform an accurate comparison between the efficacy of BEX and other reported natural products. The daily dose of BEX used in the present study was $773 \mathrm{mg} / \mathrm{kg}$ body weight for mouse, and this corresponds to $63 \mathrm{mg} / \mathrm{kg}$ body weight for human subjects $(3.8 \mathrm{~g} / \mathrm{d}$ for a $60 \mathrm{~kg}$ adult) when the body surface area normalisation method is used for an allometric dose translation $^{(26)}$.

The BEX used in the present study consists of approximately 50\% water, 20\% saccharides, $10 \%$ protein and $20 \%$ other components. The active component(s) contributing to the effects described earlier are to be further determined. The extraordinary abundance of the raw material is a major advantage of this natural product. $P$. edulis is known for its fast growth, wide geographical distribution and easy propagation. The raw materials (small branches and leaves) used for BEX production are by-products of the bamboo timber industry. Therefore, the present study suggests a potential nutraceutical application of a rich and environmentally friendly natural resource.

\section{Acknowledgements}

We thank Dr Leigh Anne Shafer who is funded by the University of Hawaii RCMI Programme for assistance with statistical analysis. The present study was supported by grant no. R21 AT003874-02 (J. P.) and R21 AT005139-01 (J. P.) from the NCCAM and ORWH, 5G12RR003061-23 from the NCRR, and 5P20 MD000173-08 from the NCMHD. The contents of this paper are solely the responsibility of the authors and do not necessarily represent the official views of the funding agencies or the National Institutes of Health. J. K. H. contributed to the MCP-1 measurement and data analysis; W. L. contributed to the animal management; M. J. B. contributed to the general consultation; J. P. contributed to the study design, data analysis and manuscript writing. The authors declare that there are no conflicts of interest.

\section{References}

1. Deshmane SL, Kremlev S, Amini S, et al. (2009) Monocyte chemoattractant protein-1 (MCP-1): an overview. J Interferon Cytokine Res 29, 313-326.

2. Sartipy P \& Loskutoff DJ (2003) Monocyte chemoattractant protein 1 in obesity and insulin resistance. Proc Natl Acad Sci U S A 100, 7265-7270. 
3. Nomura S, Shouzu A, Omoto S, et al. (2000) Significance of chemokines and activated platelets in patients with diabetes. Clin Exp Immunol 121, 437-443.

4. Kamei N, Tobe K, Suzuki R, et al. (2006) Overexpression of monocyte chemoattractant protein-1 in adipose tissues causes macrophage recruitment and insulin resistance. J Biol Chem 281, 26602-26614.

5. Fantuzzi G (2005) Adipose tissue, adipokines, and inflammation. J Allergy Clin Immunol 115, 911-919.

6. Salcedo R, Ponce ML, Young HA, et al. (2000) Human endothelial cells express CCR2 and respond to MCP-1: direct role of MCP-1 in angiogenesis and tumor progression. Blood $\mathbf{9 6}$, $34-40$.

7. Unger RH (1995) Lipotoxicity in the pathogenesis of obesitydependent NIDDM. Genetic and clinical implications. Diabetes 44, 863-870.

8. Panee J, Liu W, Lin Y, et al. (2008) A novel function of bamboo extract in relieving lipotoxicity. Phytother Res 22, 675-680.

9. Boden G, She P, Mozzoli M, et al. (2005) Free fatty acids produce insulin resistance and activate the proinflammatory nuclear factor-kappaB pathway in rat liver. Diabetes $\mathbf{5 4}$, 3458-3465.

10. Shi H, Kokoeva MV, Inouye K, et al. (2006) TLR4 links innate immunity and fatty acid-induced insulin resistance. I Clin Invest 116, 3015-3025.

11. Yu R, Kim CS, Kwon BS, et al. (2006) Mesenteric adipose tissue-derived monocyte chemoattractant protein-1 plays a crucial role in adipose tissue macrophage migration and activation in obese mice. Obesity 14, 1353-1362.

12. Heymann F, Trautwein C \& Tacke F (2009) Monocytes and macrophages as cellular targets in liver fibrosis. Inflamm Allergy Drug Targets 8, 307-318.

13. Wozniak SE, Gee LL, Wachtel MS, et al. (2009) Adipose tissue: the new endocrine organ? A review article. Dig Dis Sci 54, 1847-1856.

14. Marino M, Scuderi F, Provenzano C, et al. (2008) IL-6 regulates MCP-1, ICAM-1 and IL- 6 expression in human myoblasts. J Neuroimmunol 196, 41-48.

15. Zhang H, Chen S, Deng X, et al. (2007) The effects of Danggui-Buxue-Tang on blood lipid and expression of genes related to foam cell formation in the early stage of atherosclerosis in diabetic GK rats. Diabetes Res Clin Pract 77, 479-481.

16. Zhang HM, Chen SW, Xie CG, et al. (2006) Mechanism of Shenqi compound recipe anti-earlier diabetic artherosclerosis in GK rats. Zhongguo Zhong Yao Za Zhi 31, 1272-1276.

17. Luo P, Tan ZH, Zhang ZF, et al. (2008) Scutellarin isolated from Erigeron multiradiatus inhibits high glucose-mediated vascular inflammation. Yakugaku Zasshi 128, 1293-1299.

18. Lee YJ, Kang DG, Kim JS, et al. (2008) Buddleja officinalis inhibits high glucose-induced matrix metalloproteinase activity in human umbilical vein endothelial cells. Phytother Res 22, 1655-1659.

19. Kang MS, Hirai S, Goto T, et al. (2009) Dehydroabietic acid, a diterpene, improves diabetes and hyperlipidemia in obese diabetic KK-Ay mice. Biofactors 35, 442-448.

20. Jain SK, Rains J, Croad J, et al. (2009) Curcumin supplementation lowers TNF-alpha, IL-6, IL-8, and MCP-1 secretion in high glucose-treated cultured monocytes and blood levels of TNF-alpha, IL-6, MCP-1, glucose, and glycosylated hemoglobin in diabetic rats. Antioxid Redox Signal 11, 241-249.

21. Woo HM, Kang JH, Kawada T, et al. (2007) Active spicederived components can inhibit inflammatory responses of adipose tissue in obesity by suppressing inflammatory actions of macrophages and release of monocyte chemoattractant protein-1 from adipocytes. Life Sci 80, 926-931.

22. Abe D, Saito T, Kubo Y, et al. (2010) A fraction of unripe kiwi fruit extract regulates adipocyte differentiation and function in 3T3-L1 cells. Biofactors 36, 52-59.

23. Chacón MR, Ceperuelo-Mallafré V, Maymó-Masip E, et al. (2009) Grape-seed procyanidins modulate inflammation on human differentiated adipocytes in vitro. Cytokine $\mathbf{4 7}$, $137-142$

24. Sugimoto M, Arai H, Tamura Y, et al. (2009) Mulberry leaf ameliorates the expression profile of adipocytokines by inhibiting oxidative stress in white adipose tissue in $\mathrm{db} / \mathrm{db}$ mice. Atherosclerosis 204, 388-394.

25. Zhu J, Yong W, Wu X, et al. (2008) Anti-inflammatory effect of resveratrol on TNF-alpha-induced MCP-1 expression in adipocytes. Biochem Biophys Res Commun 369, 471-477.

26. Reagan-Shaw S, Nihal M \& Ahmad N (2008) Dose translation from animal to human studies revisited. FASEB $J \mathbf{2 2}$ $659-661$ 